\title{
The Role of Systematic Review and Meta-Analysis in Evidence-Based Medicine
}

\author{
Mohsen Saberi Isfeedvajani ${ }^{{ }^{*}}$ \\ ${ }^{1}$ Medicine, Quran and Hadith Research Center \& Department of Community Medicine, Faculty of Medicine, Baqiyatallah \\ University of Medical Sciences, Tehran, Iran \\ Corresponding Author: Mohsen Saberi Isfeedvajani, MD, Associate Professor, Medicine, Quran and Hadith Research Center \& \\ Department of Community Medicine, Faculty of Medicine, Baqiyatallah University of Medical Sciences, Tehran, Iran. Tel: +98-21- \\ 81263617, Email: drsaberihaji@gmail.com
}

Received February 22, 2018; Accepted March 1, 2018; Online Published March 20, 2018

$\mathrm{E}$ vidence-based medicine (EBM) has been defined as the "conscientious, explicit, and judicious use of current best evidence in making decisions about the care of individual patients." ${ }^{\prime \prime}$ The first important principle of EBM is hierarchy of evidence expressed as an "Evidence Pyramid."1,2 Based on this principle, the result of research is different based on study design. ${ }^{1-3}$ Systematic reviews and metaanalyses have been placed at the top of this pyramid. ${ }^{1,3,4}$ Case series/reports have been placed at the bottom, case-control and cohort studies in the middle, and randomized controlled trials (RCTs) below systematic reviews and meta-analyses on the pyramid. ${ }^{2-4}$

The Grading of Recommendations Assessment, Development and Evaluation (GRADE) Working Group modified the traditional pyramid. GRADE concluded that study design alone appears to be insufficient as a surrogate for risk of bias. In any type of study, limitations, imprecision, inconsistency, and indirectness could be factors that affect the quality of evidence derived independent of the study design. For example, randomization and blinding were not adequate in most trials. Consequently, the straight lines separating study designs in the pyramid become wavy lines. ${ }^{3,5}$

The second challenge to the "Evidence Pyramid" is a framework presented in the Journal of the American Medical Association User's Guide on systematic reviews and metaanalyses. Based on this guide, a two-step approach was defined to evaluate systematic reviews. First, the credibility of the process of a systematic review was evaluated (comprehensive literature search, rigorous study selection process). Second, the certainty in evidence based on the GRADE approach was evaluated. Consequently, systematic reviews and metaanalyses were removed from the top of the pyramid and employed as the lens through which other types of studies should be seen (i.e., appraised and applied). ${ }^{3,6}$

Clinicians and other stakeholders should consider that systematic reviews/meta-analyses have been high, moderate, low, or of very low quality although ranked as A level. In other words, a systematic review and a meta-analysis of wellconducted RCTs at a low risk of bias cannot be similar in confidence compared with a meta-analysis of observational studies (i.e., cohort, case-control, or cross-sectional) at a higher risk of bias.

\section{Conflict of Interest Disclosures}

The author declares that he has no conflicts of interest.

\section{References}

1. Chow N, Gallo L, Busse JW. Evidence-based medicine and precision medicine: Complementary approaches to clinical decision-making. Precision Clinical Medicine. 2018;1(2):60-64. doi:10.1093/pcmedi/pby009.

2. Parameswaran R, Agarwal A. Evidence-Based Surgery. In: Evidence-Based Endocrine Surgery. Singapore: Springer; 2018:310. doi:10.1007/978-981-10-1124-5.

3. Murad MH, Asi N, Alsawas M, Alahdab F. New evidence pyramid. Evid Based Med. 2016;21(4):125-127. doi:10.1136/ ebmed-2016-110401

4. Burns PB, Rohrich RJ, Chung KC. The levels of evidence and their role in evidence-based medicine. Plast Reconstr Surg. 2011;128(1):305-310. doi:10.1097/PRS.0b013e318219c171

5. Guyatt $\mathrm{GH}$, Oxman AD, Vist GE, et al. GRADE: an emerging consensus on rating quality of evidence and strength of recommendations. BMJ. 2008;336(7650):924-926. doi:10.1136/ bmj.39489.470347.AD.

6. Murad M, Montori VM, loannidis JA, et al. How to read a systematic review and meta-analysis and apply the results to patient care: Users' guides to the medical literature. JAMA. 2014;312(2):171179. doi:10.1001/jama.2014.5559. 